\title{
Identification of allergens and epitopes involved in allergy to deamidated gluten
}

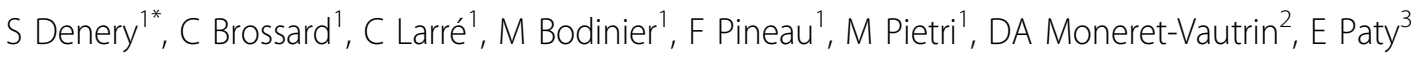 \\ From Food Allergy and Anaphylaxis Meeting (FAAM 2013) \\ Nice, France. 7-9 February 2013
}

\section{Background}

Gluten proteins can be modified by deamidation to enhance their solubility and technological applications. However severe allergic reactions have been reported after the consumption of food products containing deamidated gluten in subjects tolerant to wheat. This work aimed to characterize allergen profiles for these patients in comparison with those of patients allergic to wheat and identify IgE-binding epitopes.

\section{Methods}

Sera were obtained from 15 patients allergic to deamidated gluten (DG) and from 9 patients allergic to wheat proteins (WP). IgE-binding profiles were characterized both in ELISA and in a humanized rat basophilic leukemia (RBL) cell model. Epitopes were mapped on $\gamma$ and $\omega 2$-gliadin sequences by Pepscan and effect of glutamine/glutamic acid substitutions were studied.

\section{Results}

Compared to the heterogeneous pattern of allergens detected by IgE from patients allergic to WP, responses of patients allergic to DG were homogeneous. In ELISA, all the sera displayed IgE-binding to deamidated $\gamma$ and $\omega 2$-gliadins and deamidated total gliadins, frequently with high concentrations. These modified proteins induced RBL degranulation with most of the sera from DG-allergic patients. A consensus epitope was found on native $\gamma$ and $\omega 2$-gliadins (QPQQPFPQ); it was repeated several times in their sequences. The substitution of two or three glutamines of this epitope into glutamic acid at positions Q3 or Q4 and Q8 (QPEEPFPE) increased its recognition the best.

\section{Conclusion}

Allergy to DG is a separate entity from wheat allergy characterized by a homogeneous IgE response. Deamidated $\omega 2$-gliadins or the dominant IgE-binding epitope QPEEPFPE could be used as tools for the diagnosis of this new allergy.

\section{Disclosure of interest}

None declared.

\section{Author details}

'UR 1268 BIA Nantes, INRA, Nantes, France. ${ }^{2}$ Service d'allergologie, Centre Hospitalier Jean Monnet, Epinal, France. ${ }^{3}$ Service de Pneumologie et Allergologie Pédiatriques, Groupe Hospitalier Necker, Paris, France.

Published: 25 July 2013

\section{References}

1. Gourbeyre P, Denery-Papini S, Larré C, Gaudin J-C, Brossard C, Bodinier M: Wheat gliadins modified by deamidation are more efficient than native gliadins in inducing a th2 response in balb/c mice experimentally sensitized to wheat allergens. Mol Nutr Food Res 2012, 56(2):336-344.

2. Denery-Papini S, Bodinier M, Larré C, Brossard C, Pineau F, Triballeau S, Pietri M, Battais F, Mothes T, Paty E, Moneret-Vautrin D-A: Allergy to deamidated gluten in patients tolerant to wheat: specific epitopes linked to deamidation. Allergy 2012, 67:1023-1032.

doi:10.1186/2045-7022-3-S3-016

Cite this article as: Denery et al:: Identification of allergens and epitopes involved in allergy to deamidated gluten. Clinical and Translational Allergy 2013 3(Suppl 3):016. 